\title{
Identification of new fluorescent protein fragments for bimolecular fluorescence complementation analysis under physiological conditions
}

\author{
Y. John Shyu ${ }^{1}$, Han Liu ${ }^{1}$, Xuehong Deng ${ }^{1}$, and Chang-Deng $\mathrm{Hu}^{1,2}$ \\ ${ }^{1}$ Purdue University, West Lafayette and ${ }^{2}$ Walther Cancer Institute, Indianapolis, \\ IN, USA \\ BioTechniques 40:61-66 (January 2006) \\ doi 10.2144/000112036
}

Protein-protein interactions play a pivotal role in coordinating many cellular processes. Determination of subcellular localization of interacting proteins and visualization of $d y$ namic interactions in living cells are crucial to elucidate cellular functions of proteins. Using fluorescent proteins, we previously developed a bimolecular fluorescence complementation (BiFC) assay and a multicolor BiFC assay to visualize protein-protein interactions in living cells. However, the sensitivity of chromophore maturation of enhanced yellow fluorescent protein (YFP) to higher temperatures requires preincubation at lower temperatures prior to visualizing the BiFC signal. This could potentially limit their applications for the study of many signaling molecules. Here we report the identification of new fluorescent protein fragments derived from Venus and Cerulean for BiFC and multicolor BiFC assays under physiological culture conditions. More importantly, the newly identified combinations exhibit a 13-fold higher BiFC efficiency than originally identified fragments derived from YFP. Furthermore, the use of new combinations reduces the amount of plasmid required for transfection and shortens the incubation time, leading to a 2-fold increase in specific BiFC signals. These newly identified fluorescent protein fragments will facilitate the study of protein-protein interactions in living cells and whole animals under physiological conditions.

\section{INTRODUCTION}

Protein-protein interactions play a pivotal role in mediating signal transduction pathways and executing many cellular functions. Defining how each protein interacts with its partners under physiological conditions provides insight into the cellular functions of the protein. Several methods have been developed to study protein-protein interactions in living cells. These include mammalian two-hybrid assays (1), protein complementation assays (2-10), and fluorescent resonance energy transfer (FRET) (11-14). We previously took the protein complementation approach and used enhanced yellow fluorescent protein (EYFP) and enhanced cyan fluorescent protein (ECFP) to develop a bimolecular fluorescence complementation (BiFC) assay and a multicolor BiFC assay to directly visualize protein-protein interactions and to study the competition of multiple proteins in living cells $(15,16)$. Due to stronger complementation signal and direct readout, the $\mathrm{BiFC}$ assay has been widely accepted and used for the study of protein-protein interaction in living cells $(17,18$, and references therein). However, one limitation of EYFP is the sensitivity of chromophore maturation to higher temperatures (19). This requires preincubation of cells at lower temperatures prior to visualizing the BiFC signal $(15,16)$. Therefore, the availability of new fluorescent protein fragments that can be used under physiological conditions will undoubtedly impact the future applications of the BiFC assay. Here we report the identification of several new combinations for $\mathrm{BiFC}$ analysis under physiological conditions.

\section{MATERIALS AND METHODS}

BiFC constructs using fragments derived from three newly engineered fluorescent proteins, Venus (20), Citrine (21), and Cerulean (12) were made. Plasmids pBiFC-bJunYN155 and $\mathrm{pBiFC}-\mathrm{bFosYC155}$, as described previously (15), were used as templates. cDNAs encoding the $\mathrm{N}$ - or $\mathrm{C}$-terminal fragments of Venus, Citrine, and Cerulean were amplified by PCR and subcloned into $\mathrm{pBiFC}-\mathrm{bJunYN} 155$ and pBiFC-bFosYC155 to replace those encoding YN155 and YC155 (Figure 1, A and B). To facilitate the detection of their expression, all N-terminal fragments fused to bJun have a FLAG ${ }^{\circledR}$ tag at their $\mathrm{N}$ termini, and $\mathrm{C}$-terminal fragments fused to bFos have a hemagglutin (HA) tag at their $\mathrm{N}$ termini.

COS-1 cells were cultured in Dulbecco's modified Eagle medium (Hyclone, Logan, UT, USA) supplemented with $10 \%$ fetal bovine serum with penicillin and streptomycin in 12 -well plates at $37^{\circ} \mathrm{C}$. The indicated amounts of plasmid encoding bJun or bFos fusion proteins were transfected into COS- 1 cells using FuGENE ${ }^{\circledR} 6$ (Roche Applied Science, Indianapolis, IN, USA). For ratio analysis, plasmids encoding ECFP or EYFP were cotransfected as an internal control to measure the BiFC efficiency of fragments derived from YFP or CFP variants, respectively. Images of transfected cells $24 \mathrm{~h}$ after transfection were captured using a charge-coupled device (CCD) camera mounted on a TE2000-U inverted fluorescence microscope (Nikon, Melville, NY, USA) with JP4 filters (Chroma, Rockingham, VT, USA). The intensity of more than 100 cells was individually quantified using an automated intensity recognition feature of Metamorph II (Universal Imaging, Downingtown, PA, USA). YFP/CFP ratio analysis was similarly performed as reported previously (15). The median of YFP/CFP ratios was used for comparison of the BiFC efficiency among YFP variants and determination of BiFC specificity, since the distribution of the raw data was highly skewed (15). Since $7.8 \%$ of the BiFC signal derived from N173 of Venus paired with C155 of ECFP was 
read in the CFP channel, we corrected this signal crosstalk by multiplying original CFP images with a correction coefficient. Briefly, cells transfected with plasmids encoding N173 of Venus fused to bJun and C155 of ECFP fused to bFos were used as a control to determine the correction coefficient.

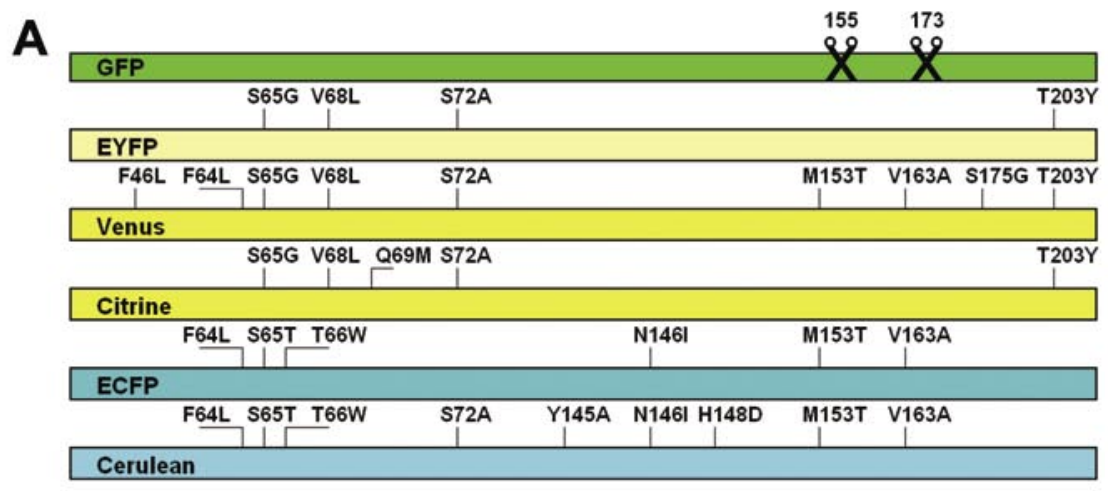

B

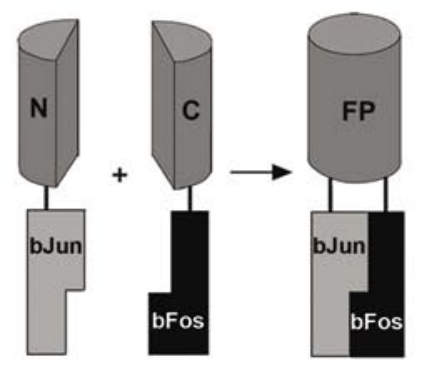

D

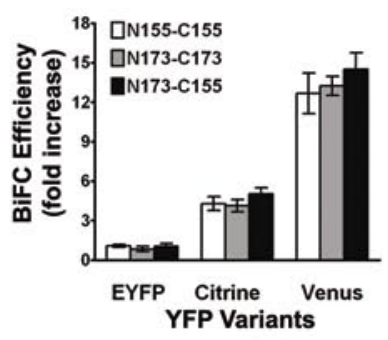

E

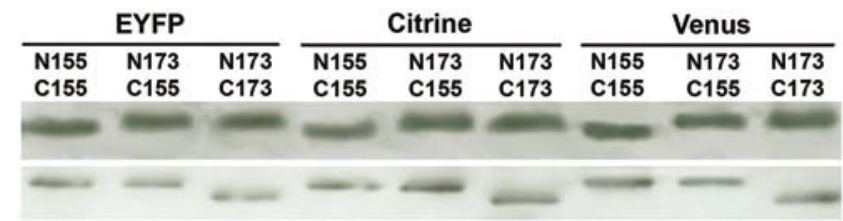

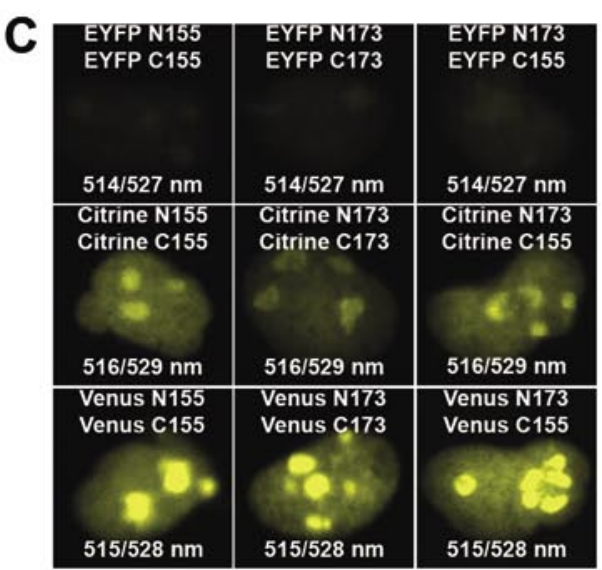

Anti-FLAG

Anti-HA
Figure 1. Identification of new fluorescent protein fragments for bimolecular fluorescence complementation (BiFC) analysis under physiological conditions. (A) View of enhanced green fluorescent protein (EGFP) and its variants. The indicated residues are substitutions from GFP, and the positions of truncation in fluorescent proteins are indicated with a scissors symbol. (B) View of BiFC principle. $\mathrm{N}$ - and C-terminal fragments of fluorescent proteins were fused to bJun and bFos, respectively. The interaction between bJun and bFos brings $\mathrm{N}$ - and C-terminal fragments in proximity to reconstitute an intact fluorescent protein (FP). (C) Fluorescent images of COS-1 cells transfected with $0.125 \mu \mathrm{g}$ plasmid encoding bJun or bFos fused to the fluorescent protein fragments indicated in each panel. The number indicates the maxima of excitation and emission for each BiFC complex. (D) Quantification of BiFC efficiency among yellow fluorescent protein (YFP) mutants. The BiFC efficiency was determined as described in the main text. The fold increase of median ratios plus standard deviation was determined when compared with N155-C155 of enhanced YFP (EYFP). (E) Immunoblot analysis of fusion proteins. Transfected cells expressing the indicated fusion proteins from Figure $1 \mathrm{C}$ were harvested for the detection of fusion proteins using anti-FLAG and anti-HA antibodies. ECFP, enhanced cyan fluorescent protein.
Fluorescent images were captured with both YFP and CFP filters, and the correction coefficient was calculated as described for FRET (22). The original $\mathrm{CFP}$ images in the multicolor $\mathrm{BiFC}$ experiments were multiplied by the correction coefficient using the FRET function provided in Metamorph II, and a representative of the corrected CFP images was presented here (see Figure 3B).

To determine the excitation and emission maxima of each new BiFC complex, COS-1 cells were transfected with $0.25 \mu \mathrm{g}$ of each plasmid encoding the fusion proteins and incubated at $37^{\circ} \mathrm{C}$ for $24 \mathrm{~h}$. Harvested cells were resuspended in $1 \times$ phosphate-buffered saline (PBS) buffer (Hyclone), and FluoroMax ${ }^{\circledR} 2$ was used in conjunction with the Discovery Scan feature of DataMax for Windows (Horiba Jobin Yvon, Edison, NJ, USA) to determine the excitation and emission maxima of each BiFC complex.

To determine BiFC specificity, cells were cotransfected with various amounts of plasmid encoding each fusion protein in combination with $50 \mathrm{ng}$ plasmid encoding ECFP. The median of YFP/CFP ratios was calculated from more than 100 cells. The specificity of BiFC represents the fluorescent signals derived from the reconstituted fluorescent proteins driven by specific bFos-bJun interactions. For the purpose of comparison among different groups transfected with different amounts of plasmid, the BiFC specificity was calculated as fold increase by dividing the median ratio derived from cells coexpressing bFos fusions paired with bJun fusions by the median ratio derived from cells coexpressing bFos( $\Delta \mathrm{Zip})$ fusions paired with bJun fusions. Note that $\mathrm{bFos}(\Delta \mathrm{Zip})$ is a dimerization-deficient mutant and was paired with bJun as a background control $(15,16,18)$.

To determine the expression level of various fusion proteins, transfected cells were harvested after images were captured and lysed in $100 \mu \mathrm{L} 1 \times$ Laemmli sample loading buffer. The proteins were resolved by $10 \%$ sodium dodecyl sulfate polyacrylamide gel electrophoresis (SDS-PAGE), followed by the transfer to a nitrocellulose filter. The expression of each fusion protein 
was determined using antibodies against the FLAG or HA epitopes (Sigma, St. Louis, MO, USA).

\section{RESULTS AND DISCUSSION}

To identify new fluorescent protein fragments for BiFC analysis under physiological conditions, we examined

A

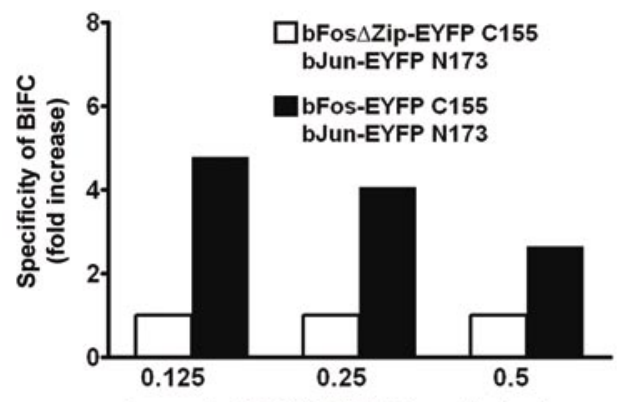

Amount of EYFP BiFC Plasmids $(\mu \mathrm{g})$

B

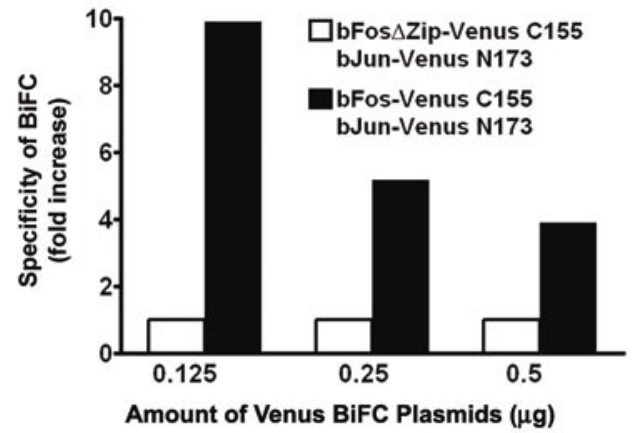

C

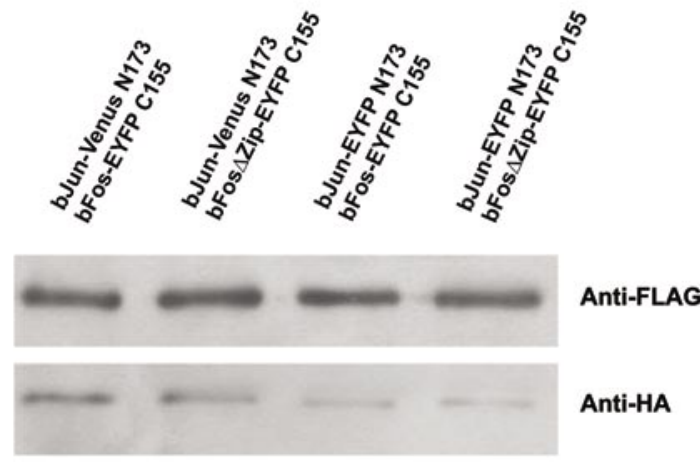

Figure 2. Comparison of the specificity of bimolecular fluorescence complementation (BiFC) signal using enhanced yellow fluorescent protein (EYFP) and Venus fragments. Cells were transfected with the indicated amount of each plasmid, and the specificity of BiFC was determined as described in the main text. (A) Effect of different amounts of plasmids on the specificity of BiFC using EYFP fragments. (B) Effect of different amounts of plasmids on the specificity of BiFC using Venus fragments. (C) Immunoblot analysis of fusion proteins. Transfected cells expressing the indicated fusion proteins from panels A and B were harvested for the detection of fusion proteins using anti-FLAG and anti-HA antibodies.
BiFC efficiency using Citrine and engineered to be less sensitive to the errom thents derived from D155 or D173 can support BiFC when fused to the basic region leucine zipper domain of c-Jun (bJun) and the basic region leucine zipper domain of c-Fos (bFos) (Figure 1, A and $\mathrm{B})(15,16)$, we similarly truncated Citrine and Venus at these two positions and fused the $\mathrm{N}$ - and C-terminal fragments to bJun and bFos, respectively (Figure 1B). Coexpression of these fusion proteins in COS-1 cells resulted in fluorescence complementation in all combinations (Figure 1C). Quantification of the BiFC efficiency revealed that all three combinations of Venus showed a 12to 15 -fold increase and Citrine showed a 4- to 5-fold increase when compared with EYFP (Figure 1D). All combinations of fusion proteins were comparably expressed (Figure 1E). These experiments were carried out at $37^{\circ} \mathrm{C}$, and images were captured $24 \mathrm{~h}$ posttransfection. Preincubation of cells at $30^{\circ} \mathrm{C}$ for $4 \mathrm{~h}$ prior to image capturing significantly increased the BiFC signal for EYFP fragments, whereas the BiFC signals derived from Citrine and Venus fragments were not significantly affected (data not shown). This confirms that the chromophore maturation of Citrine and Venus is indeed less sensitive to higher temperatures. Nevertheless, the average fluorescence intensity derived from EYFP fragments was still only $64 \%$ and $40 \%$ of that derived from Citrine and Venus, respectively.

To determine whether the newly identified fluorescent protein fragments affect the specificity of the BiFC signal, we used bFos( $\Delta \mathrm{Zip})$, carrying a small deletion in the Zip region to eliminate specific dimerization with bJun (15), as a background control to compare the BiFC efficiency between wild-type bFos and this mutant bFos when they were paired with bJun fusion proteins $(15,16,18)$. As shown in Figure 2A, when fragments derived from EYFP were used, the BiFC signal derived from wild-type bFos was 3- to 5-fold higher than those derived from bFos $\Delta$ Zip within the range of amount of plasmids examined. Lower amount of plasmids for transfection showed relatively higher specificity. Similar tendency was observed when fragments derived from Venus were used (Figure 2B). However, the use of Venus BiFC plasmids exhibited higher specificity when compared with EYFP. In particular, the use of $0.125 \mu \mathrm{g}$ plasmids encoding fusion proteins with Venus fragments showed 2-fold higher specificity than the use of EYFP fragments. Comparable expression of the fusion proteins was confirmed by immunoblot analysis (Figure 2C). When the fulllength c-Fos and c-Jun were examined using the Venus fragments, we observed very bright BiFC signals $12 \mathrm{~h}$ after transfection, whereas the use of EYFP fragments required 2 days incubation at $37^{\circ} \mathrm{C}$ plus at least $2 \mathrm{~h}$ preincubation at $30^{\circ} \mathrm{C}$ (data not shown). These results demonstrate that the use of Venus fragments for BiFC analysis not only eliminates the preincubation at lower temperatures, but also shows higher specificity and requires lower amount of plasmids for transfection, plus a shorter incubation time.

Protein-protein interaction is the foundation of signaling networks in cells. Each protein has a few, or even a large number of, alternative interaction partners. These alternative interactions are often selectively induced in response to specific signals. 
Alternatively, several interaction partners that coexist in the same subcellular compartment may compete for binding to shared interaction partners. Therefore, visualization of multiple interactions in the same cell provides a unique way to study protein interaction networks. Since green fluorescent protein (GFP) variants have virtually identical structure, and the chromophore is formed by three residues at positions 65, 66, and 67 (19), we previously examined all possible combinations among fragments derived from four GFP spectral variants and identified several combinations that showed better spectral resolution and supported the fluorescence complementation (16). The best combination

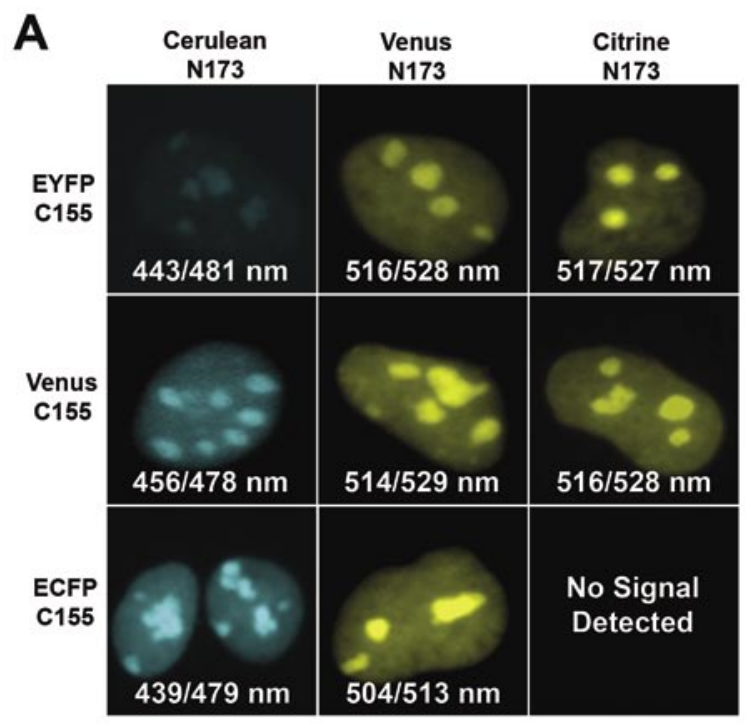

B

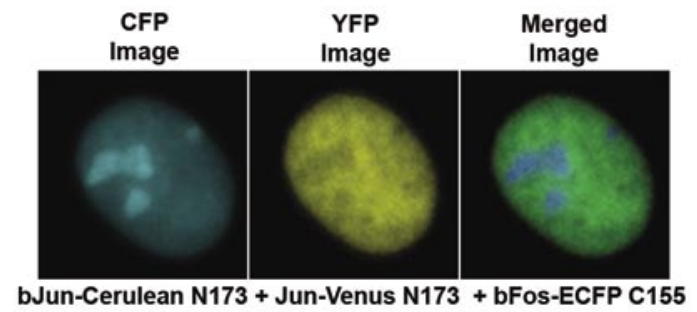

Figure 3. Identification of new combinations for multicolor bimolecular fluorescence complementation (BiFC) analysis. (A) Fluorescent images of COS-1 cells transfected with $0.125 \mu \mathrm{g}$ plasmids encoding bJun or bFos fused to the fluorescent protein fragments indicated. The number indicates the maxima of excitation and emission for each BiFC complex. (B) Visualization of differential subnuclear localization of bJun-bFos and Jun-bFos. Plasmids encoding the indicated fusion proteins were cotransfected into COS-1 cells, and images were taken using cyan fluorescent protein (CFP) and yellow fluorescent protein (YFP) filters. Note that the CFP image was corrected as described in the main text. identified previously for multicolor BiFC analysis was the C-terminal fragment of ECFP (ECFP C155) paired with the N-terminal fragments of EYFP (EYFP N173) and ECFP (ECFP N173) (16,23). Recently, it has been reported Cerulean, an improved ECFP, (12). This prompted us to explore the BiFC combination may be identified in conjunction with the newly identified fluorescent protein fragments described above. The C-terminal sequences of EYFP and Citrine are identical, as are those of ECFP and Cerulean (Figure 1A). We compared the complementation efficiency of the C-terminal fragments comprised of residues 155-238 (C155) derived from EYFP, Venus, and ECFP with the N-terminal fragments comprised of residues 1172 (N173) of Cerulean, Citrine, and Venus. C155 of ECFP showed higher complementation efficiency and lower signal bleed-through with N173 derived from Cerulean and Venus than any other combinations (Figure $3 \mathrm{~A}$ and data not shown). Interestingly, N173 of Citrine complemented with C155 of EYFP and Venus, but did not complement with $\mathrm{C} 155$ of ECFP. The only difference among fragments $\mathrm{C} 155$ of EYFP, Venus, and ECFP is T203Y (Figure 1A), suggesting the importance of Y203 in fragment complementation of fluorescent proteins. We previously observed that the bZip domain dimers of all bZip proteins were predominantly localized in the nucleoli, whereas the complexes formed by a bZip domain and a fulllength bZip protein were predominantly localized to the nucleoplasm (15). This distinct subcellular localization was visualized simultaneously using our multicolor BiFC assay (16). To validate the usefulness of the newly identified combination for multicolor BiFC assay, we examined the subnuclear localization of bFosbJun heterodimers and bFos-Jun heterodimers in the same cell (16). When this combination was used, bright fluorescent signals derived from bFos-bJun heterodimers were predominantly localized in the nucleoli (Figure 3B). In contrast, bFos-Jun heterodimers were distinctively localized in the nucleoplasm. Again, transfected cells were only incubated at $37^{\circ} \mathrm{C}$ for $24 \mathrm{~h}$.

In summary, we have identified Venus as the most useful yellow fluorescent protein for BiFC analysis of protein-protein interactions under physiological culture conditions. Furthermore, a new combination between C155 of ECFP and N173 of Cerulean and Venus has proven to be the best combination for multicolor BiFC analysis. The newly identified fragments offer two significant advantages over the previously reported $\mathrm{BiFC}$ constructs using EYFP and ECFP. First, the new combinations allow for lower amounts of plasmid for transfection and shorter incubation time, two recommended strategies to reduce nonspecific signals (18). Second, the new combinations eliminate the need for preincubaton of cells at lower temperatures. Because a decrease in temperature itself is a stressful condition, the new combinations should be particularly useful for the study of signaling molecules involved in the stress response. These improvements lead to the increase of both the BiFC signal and the specificity. It is also very likely that the new combinations will be suitable for visualization of protein-protein interactions in whole animals. However, compared with other protein fragment complementation assays using enzymes, such as luciferase (9), $\beta$-galactosidase (2), and $\beta$-lactamase $(4,5)$, the BiFC assay does not provide signal amplification. In addition, it remains to be determined whether the BiFC assay can be used to visualize transient and inducible interactions (e.g., within minutes) or dynamic interaction in cells, since the chromophore maturation of fluorescent 
proteins requires at least $30 \mathrm{~min}$ (19), and the BiFC complex is irreversible in vitro (15).

One interesting finding in this and our previous BiFC analysis of GFP spectral variants is the unpredictability of fluorescence complementation. We initially thought that the truncation at positions 155 and 173 would be applicable to all GFP variants. However, neither of these positions worked with EGFP (16). Interestingly, N-terminal fragments truncated at either site complemented with the C-terminal fragments derived from EYFP. The only difference between the C-terminal fragments of EYFP and EGFP is the residue at position 203 (Figure 1A). In the case of EYFP, Y203 was used in place of T203. This substitution allows a $\pi-\pi$ interaction with the chromophore phenol ring (24), suggesting that this interaction not only changes the spectra of the protein but also contributes to the reconstitution of the $\beta$-barrel structure. Similar to this Y203, our present analysis suggests that the position 69 also is critical to the fluorescence complementation. Fragments derived from Citrine and Venus all complemented with each other and showed higher BiFC efficiency than fragments derived from EYFP when cells were cultured at $37^{\circ} \mathrm{C}$. This is due to the introduction of the substituted residues Q69M for Citrine and F46L for Venus $(20,21)$. These substitutions are thought to facilitate chromophore maturation by increasing protein folding at $37^{\circ} \mathrm{C}$. In addition to these two critical substitutions in Citrine and Venus, additional substitutions (F64L, M153T, V163A, and $\mathrm{S} 175 \mathrm{G}$ ) in Venus are also known to improve protein folding at $37^{\circ} \mathrm{C}$ $(19,20)$, and these changes might contribute to higher BiFC efficiency for Venus over other YFP variants. Surprisingly, N173 of Citrine did not complement with C155 of ECFP, but N173 of EYFP did (Figure 3A). Since Q69M is the only difference between EYFP and Citrine, this observation suggests that the residue at position 69 not only affects the chromophore maturation but also contributes to the fragment complementation. However, N173 of Citrine complemented well with C155 of EYFP, Venus, and Citrine. Again, this is probably due to the presence of Y203, which might compensate for the presence of M69 in N173 of Citrine. Since the chromophore of fluorescent proteins must be protected from the environment (25), it is important that the reassembly of fluorescent protein fragments is precise. It is therefore likely that complementation of fluorescent protein fragments is determined by a combination of residues from both $\mathrm{N}$ and C-terminal fragments. Consistent with this notion, truncation at neither position 155 nor position 173 of EGFP supports the BiFC in our case (16), whereas truncation at position 157 of EGFP allows the resulting fragments to complement (7). Structural analysis of these reconstituted $\mathrm{BiFC}$ complexes will be necessary to provide insight into the mechanisms by which BiFC works. Regardless of the mechanisms, the newly identified fluorescent protein fragments reported here for $\mathrm{BiFC}$ analysis will be useful for the study of protein-protein interactions in living cells.

\section{ACKNOWLEDGMENTS}

We would like to thank Dr. Tom Kerppola for his critical reading and comments on the manuscript, as well as Drs. Atsushi Miyawaki and David Piston for kindly providing cDNAs of Venus and Cerulean, and the members of the Hu laboratory for helpful discussions. This work was partly supported by the Purdue Cancer Center (NCI-P30CA23168), the Indina Elks Charities, Inc., the Walther Cancer Institute, and a grant from National Science Foundation (0420634-MCB) to C.D.H.

\section{COMPETING INTERESTS STATEMENT}

The authors declare no competing interests.

\section{REFERENCES}

1. Luo, Y., A. Batalao, H. Zhou, and L. Zhu. 1997. Mammalian two-hybrid system: a complementary approach to the yeast two-hybrid system. BioTechniques 22:350-352.
2. Rossi, F.M., B.T. Blakely, C.A. Charlton, and H.M. Blau. 2000. Monitoring proteinprotein interactions in live mammalian cells by beta-galactosidase complementation. Methods Enzymol. 328:231-251.

3. Johnsson, N. and A. Varshavsky. 1994. Split ubiquitin as a sensor of protein interactions in vivo. Proc. Natl. Acad. Sci. USA 91:1034010344.

4. Wehrman, T., B. Kleaveland, J.H. Her, R.F. Balint, and H.M. Blau. 2002. Protein-protein interactions monitored in mammalian cells via complementation of beta-lactamase enzyme fragments. Proc. Natl. Acad. Sci. USA 99:3469-3474.

5. Galarneau, A., M. Primeau, L.E. Trudeau, and S.W. Michnick. 2002. Beta-lactamase protein fragment complementation assays as in vivo and in vitro sensors of protein protein interactions. Nat. Biotechnol. 20:619-622.

6. Paulmurugan, R. and S.S. Gambhir. 2005. Firefly luciferase enzyme fragment complementation for imaging in cells and living animals. Anal. Chem. 77:1295-1302.

7. Ghosh, I., A.D. Hamilton, and L. Regan. 2000. Antiparallel leucine zipper-directed protein reassembly: applications to the green fluorescent protein. J. Am. Chem. Soc. 122:5658-5659.

8. Michnick, S.W., I. Remy, F.X. CampbellValois, A. Vallee-Belisle, and J.N. Pelletier 2000. Detection of protein-protein interactions by protein fragment complementation strategies. Methods Enzymol. 328:208-230.

9. Luker, K.E. and D. Piwnica-Worms. 2004 Optimizing luciferase protein fragment complementation for bioluminescent imaging of protein-protein interactions in live cells and animals. Methods Enzymol. 385:349-360.

10.Rackham, O. and C.M. Brown. 2004 Visualization of RNA-protein interactions in living cells: FMRP and IMP1 interact on mRNAs. EMBO J. 23:3346-3355

11.Miyawaki, A., J. Llopis, R. Heim, J.M. McCaffery, J.A. Adams, M. Ikura, and R.Y. Tsien. 1997. Fluorescent indicators for $\mathrm{Ca}^{2+}$ based on green fluorescent proteins and calmodulin. Nature 388:882-887.

12.Rizzo, M.A., G.H. Springer, B. Granada, and D.W. Piston. 2004. An improved cyan fluorescent protein variant useful for FRET. Nat. Biotechnol. 22:445-449.

13.Pollok, B.A. and R. Heim. 1999. Using GFP in FRET-based applications. Trends Cell Biol. 9:57-60.

14.Periasamy, A. and R.N. Day. 1999 Visualizing protein interactions in living cells using digitized GFP imaging and FRET microscopy. Methods Cell Biol. 58:293-314.

15.Hu, C.D., Y. Chinenov, and T.K. Kerppola. 2002. Visualization of interactions among bZIP and Rel family proteins in living cells using bimolecular fluorescence complementation. Mol. Cell 9:789-798.

16.Hu, C.D. and T.K. Kerppola. 2003. Simultaneous visualization of multiple protein interactions in living cells using multicolor fluorescence complementation analysis. Nat. Biotechnol. 21:539-545.

17.Wilson, C.G., T.J. Magliery, and L. Regan. 2004. Detecting protein-protein interactions 
with GFP-fragment reassembly. Nat. Methods 1:255-262.

18.Hu, C.D., A. Grinberg, and T. Kerppola. 2005. Visualization of protein interaction in living cells using bimolecular fluorescence complementation $(\mathrm{BiFC})$ analysis, p. 21.3.121.3.21. In J.S. Bonifacino, M. Dasso, J.B. Harford, J. Lippincott-Schwartz, and K.M. Yamada (Eds.), Current Protocol in Cell Biology. John Wiley \& Sons, New York.

19.Tsien, R.Y. 1998. The green fluorescent protein. Annu. Rev. Biochem. 67:509-544.

20.Nagai, T., K. Ibata, E.S. Park, M. Kubota, K. Mikoshiba, and A. Miyawaki. 2002. A variant of yellow fluorescent protein with fast and efficient maturation for cell-biological applications. Nat. Biotechnol. 20:87-90.

21.Griesbeck, O., G.S. Baird, R.E. Campbell, D.A. Zacharias, and R.Y. Tsien. 2001. Reducing the environmental sensitivity of yellow fluorescent protein. Mechanism and applications. J. Biol. Chem. 276:29188-29194.

22.Gordon, G.W., G. Berry, X.H. Liang, B. Levine, and B. Herman. 1998. Quantitative fluorescence resonance energy transfer measurements using fluorescence microscopy. Biophys. J. 74:2702-2713.

23.Grinberg, A.V., C.D. Hu, and T.K. Kerppola. 2004. Visualization of Myc/Max/ Mad family dimers and the competition for dimerization in living cells. Mol. Cell. Biol. 24:4294-4308.

24.Wachter, R.M., M.A. Elsliger, K. Kallio, G.T. Hanson, and S.J. Remington. 1998. Structural basis of spectral shifts in the yellow-emission variants of green fluorescent protein. Structure 6:1267-1277.

25.Ormo, M., A.B. Cubitt, K. Kallio, L.A. Gross, R.Y. Tsien, and S.J. Remington. 1996. Crystal structure of the Aequorea victoria green fluorescent protein. Science 273:1392-1395.

Received 11 July 2005; accepted 18 August 2005.

Address correspondence to Chang-Deng $\mathrm{Hu}$, Department of Medicinal Chemistry \& Molecular Pharmacology and the Purdue Cancer Center, Purdue University, West Lafayette, IN 47907, USA. e-mail: cdhu@pharmacy.purdue.edu

To purchase reprints

of this article, contact

Reprints@BioTechniques.com 